\title{
Thrombus straddling a patent foramen ovale
}

\section{Trombo a cavaliere di una pervietà del forame ovale}

\author{
Costantina Prota, Rodolfo Citro, Angelo Silverio, Roberto Ascoli, \\ Corrado Naddeo, Giovanni Vitale, Federico Piscione
}

\begin{abstract}
Thrombus straddling a patent foramen ovale. C. Prota, R. Citro, A. Silverio, R. Ascoli, C. Naddeo, G. Vitale, F. Piscione.

We present a case of a 67-year-old male with pulmonary embolism. Transesophageal echocardiography (TEE) showed the presence of a mobile thrombus straddling the patent foramen ovale (PFO) and prolapsing into both atria. Treatment with heparin was started. Five days after
\end{abstract}

admission, repeat TEE revealed a reduction in thrombus dimensions, so anticoagulation therapy was continued. Eleven days after admission, TEE showed complete disappearance of the thrombus.

Keywords: Thrombosis, Patent Forame Ovale, Pulmonary Embolism.

Monaldi Arch Chest Dis 2013; 80: 137-139.

Heart Department, University Hospital "San Giovanni di Dio e Ruggi d'Aragona”, Salerno, Italy.

Corresponding author: Rodolfo Citro, MD FESC; University Hospital "San Giovanni di Dio e Ruggi d'Aragona", Heart Tower room 810. Largo Città di Ippocrate, I-84131 Salerno, Italy; Phone: +39 089 673377, Mobile: +39 3473570880 , E-mail address: rodolfocitro@gmail.com

\section{Case Report}

A 67-year-old male with a history of polycythemia was admitted to our hospital for increasing dyspnea on exertion. His blood pressure was 110/80 $\mathrm{mmHg}$; the ECG showed sinus tachycardia (heart rate $120 \mathrm{bpm}$ ) and incomplete right bundle branch block; troponin I $(1.10 \mathrm{ng} / \mathrm{ml} ;$ n.v. $0-0.03 \mathrm{ng} / \mathrm{ml})$ and D-dimer (822 ng/dl; n.v. $<500 \mathrm{ng} / \mathrm{dl}$ ) were elevated.

At transthoracic echocardiography, the right cardiac chambers were enlarged and pulmonary artery pressure was elevated $(55 \mathrm{mmHg})$. Chest spiral computed tomography revealed a thrombus partially occluding the bilateral pulmonary arteries, which confirmed the suspicion of pulmonary embolism.
Treatment with heparin was started.

Transesophageal echocardiography (TEE) showed the presence of a mobile thrombus straddling the patent foramen ovale (PFO) and prolapsing into both atria (Fig. 1). A lung perfusion scintigraphy showed areas of perfusion deficit. No deep vein thrombosis was detected at lower extremity Doppler ultrasound examination.

Five days after admission, repeat TEE revealed a reduction in thrombus dimensions (Fig. 2). Anticoagulation therapy with heparin plus acenocoumarol and then with acenocoumarol alone was maintained until the INR was 2.5. Eleven days after admission, TEE showed complete disappearance of the thrombus (Fig. 3).

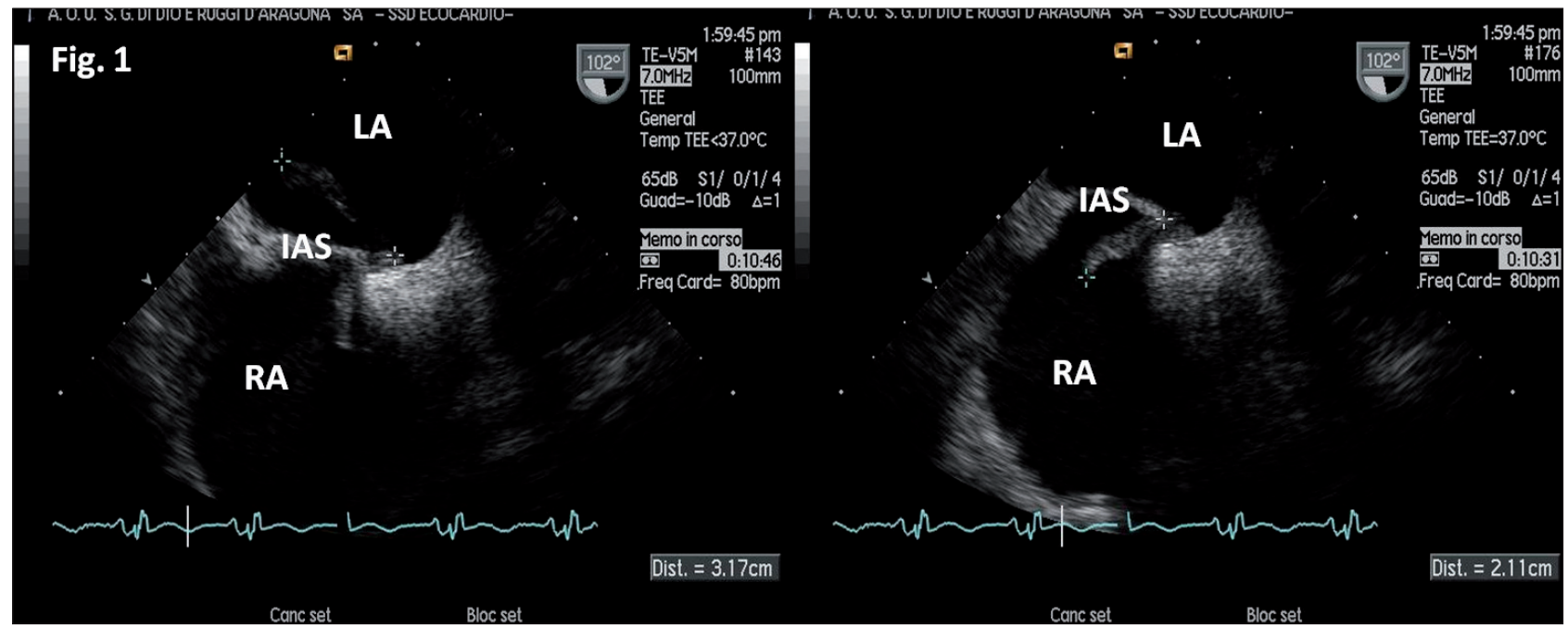

Figure 1. - Transesophageal echocardiography shows a large mobile thrombus straddling the patent foramen ovale and extending from the right to the left atrium, with a club-like morphology. Most of the thrombus was in the left atrium $(31.7 \times 8 \mathrm{~mm})$, and the remaining in the right atrium $(21.1 \times 8 \mathrm{~mm})$. LA, left atrium; RA, right atrium; IAS, interatrial septum; Ao, aorta. 


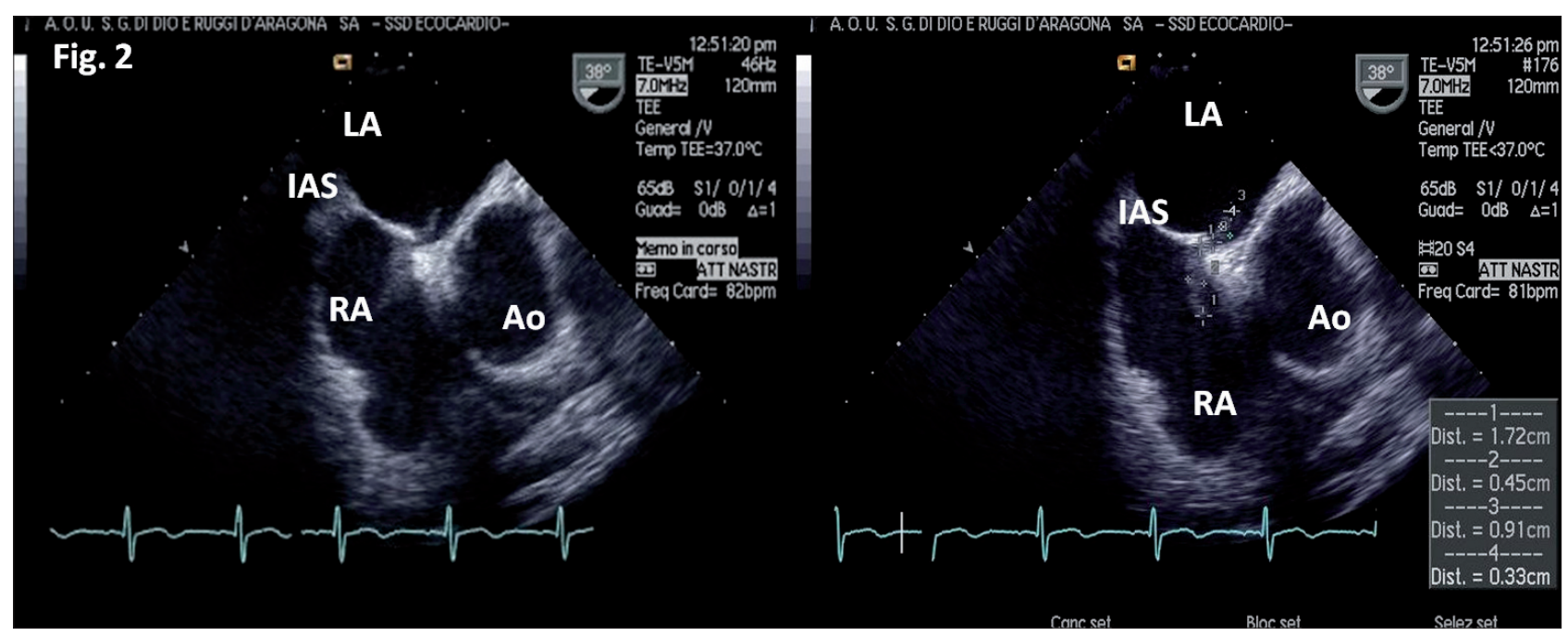

Figure 2. - A second transesophageal echocardiography revealed the decrease of dimensions of the thrombus particularly in left atrium ( $9.1 \mathrm{x} 3.3$ $\mathrm{mm})$ but also in right atrium $(17.2 \times 4.5 \mathrm{~mm})$. LA, left atrium; RA, right atrium; IAS, interatrial septum; Ao, aorta.

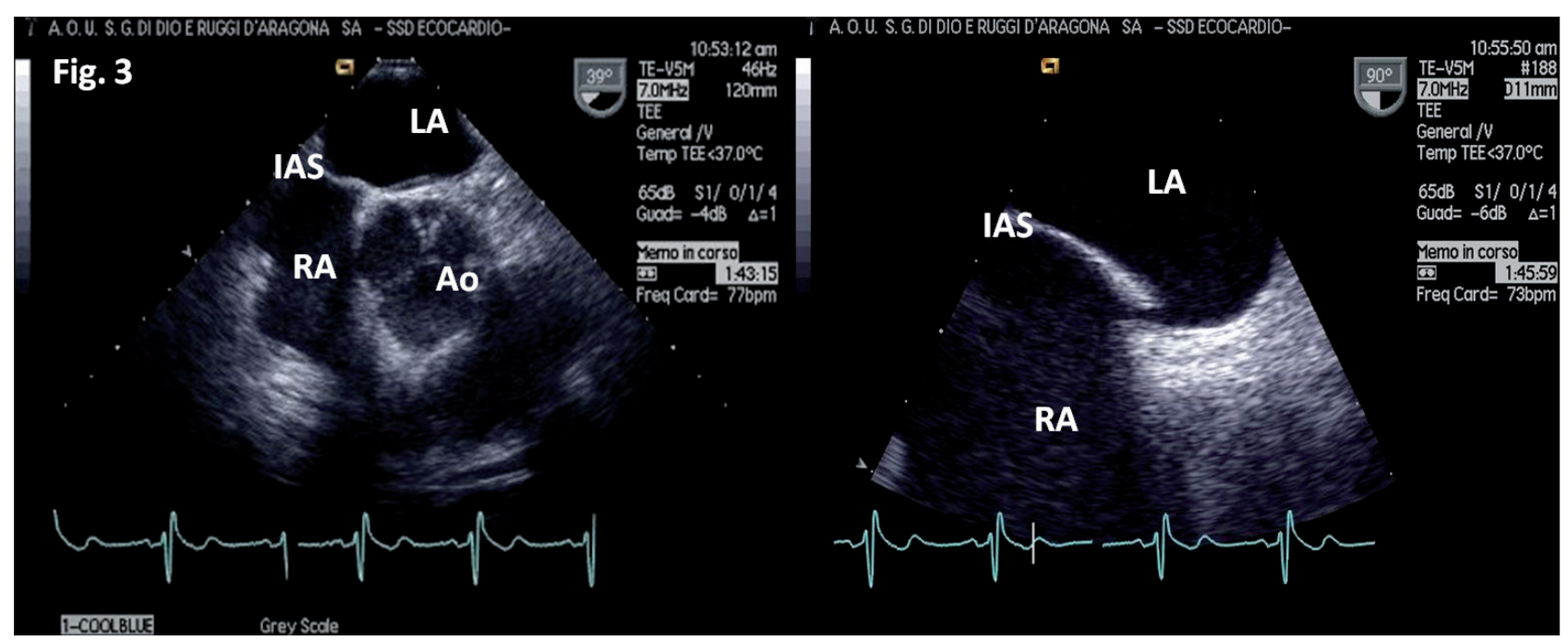

Figure 3. - A third transesophageal echocardiography revealed no thrombus after anticoagulation therapy. LA, left atrium; RA, right atrium; IAS, interatrial septum; Ao, aorta.

The patient was discharged after 17 days from admission on anticoagulation therapy. At 1-year follow-up, no thromboembolic events were reported. Moreover, JAK2-V617F mutation has been investigated because of the suspect of polycythemia vera but it was absent.

The patient is now in close follow-up in order to evaluate the possible percutaneous closure of patent foramen ovale once anticoagulation therapy will be discontinued.

A thrombus straddling a PFO is a rare event. It can be associated with pulmonary embolism (more frequently) or systemic paradoxical embolism. Anticoagulation therapy is the first-line approach to this condition. It usually has a favorable outcome ${ }^{1}$, as demonstrated in our case. However, heparin alone may be ineffective because it has no direct action on clot lysis ${ }^{2}$. Surgical treatment may be a valuable option in case of unresolved thrombus 3 . Owing to the risk of thrombus fragmentation, thrombolysis is associated with high mortality and should be used with caution $[1,4]$.

\section{Riassunto}

Presentiamo il caso di un uomo di 67 anni affetto da embolia polmonare.

L'ecocardiografia transesofagea (ETE) mostrava l'immagine di un trombo mobile a cavaliere del forame ovale pervio e prolassante in entrambi gli atri. Fu sottoposto a trattamento anticoagulante con eparina. Cinque giorni dopo l' ingresso in ospedale, una seconda ETE mostrava una consistente riduzione delle dimensioni del trombo; fu deciso quindi di continuare il trattamento anticoagulante. Undici giorni dopo, una terza ETE mostrava la completa scomparsa del trombo.

\section{ABBREVIATIONS}

TEE $=$ Transesophageal echocardiography

PFO $=$ Patent foramen ovale

ECG $=$ elettrocardiogram

No conflict of interest to declare. 


\section{References}

1. Fauveau E, Cohen A, Bonnet N, et al. Surgical or medical treatment for thrombus straddling the patent foramen ovale: impending paradoxical embolism? Report of four clinical cases and literature review. Arch Cardiovasc Dis 2008; 101: 637-644.

2. Bonvini RF, Robert-Ebadi H, Fontana P, et al. Impending paradoxical embolism. When and how to treat? Ann Cardiol Angeiol 2008; 57: 234-237.
3. Citro R, Panza A, Bottiglieri G, et al. Surgical treatment of impending paradoxical embolization associated with pulmonary embolism in a patient with heterozygosis of factor V Leiden. J Cardiovasc Med 2010 Jul 15 [Epub ahead of print].

4. Myers PO, Bounameaux H, Panos A, et al. Impending paradoxical embolism: systematic review of prognostic factors and treatment. Chest 2010; 137: 164-170. 